\title{
The influence of tobacco smoke/nicotine on CYP2A expression in human and African green monkey lungs
}

\author{
Yuan Gao ${ }^{1}$, Sharon Miksys ${ }^{1}$, Roberta Palmour ${ }^{2}$, and Rachel Tyndale ${ }^{1}$ \\ ${ }^{1}$ University of Toronto \\ ${ }^{2}$ McGill University
}

June 22, 2020

\begin{abstract}
BACKGROUND AND PURPOSE CYP2A enzymes metabolically inactivate nicotine and activate tobacco-derived procarcinogens (e.g. NNK, 4-(methylnitrosamino)-1-(3-pyridyl)-1-butanone). Smoking decreases nicotine clearance, and chronic nicotine reduces hepatic CYP2A activity. However, little is known about the impact of smoking or nicotine on the expression of CYP2A in the lung. We investigated 1) the levels of human lung CYP2A mRNA in smokers versus non-smokers and 2) the impact of daily nicotine treatment on lung CYP2A protein levels in African Green Monkeys (AGM). EXPERIMENTAL APPROACH Lung CYP2A13, CYP2A6 and CYP2A7 (and CYP1A2) mRNA levels in smokers and non-smokers were assessed in Gene Expression Omnibus (GEO) datasets (GSE40364, GSE11874 and GSE103845). The impact of chronic twice daily subcutaneous nicotine at two doses $(0.3$ and $0.5 \mathrm{mg} \mathrm{kg}-1)$, versus vehicle, on lung CYP2A protein levels was assessed. The impact of ethanol self-administration was also investigated, with and without nicotine treatment. KEY RESULTS Smokers, versus non-smokers, had significantly lower levels of lung CYP2A13, CYP2A6 and CYP2A7 (and increased CYP1A2) mRNA in both GEO datasets. Nicotine treatment, at both doses, significantly decreased AGM lung CYP2A protein. Ethanol self-administration had no effect on AGM lung CYP2A protein, and there was no interaction between ethanol and nicotine. CONCLUSIONS AND IMPLICATIONS Smoking was associated with a reduction in human lung CYP2A13, CYP2A6, and CYP2A7 mRNA, consistent with the role of nicotine treatment in reducing AGM lung CYP2A protein. This regulation by smoking/nicotine will increase interindividual variation in lung CYP2A levels that may impact the localized metabolism of inhaled drugs and tobacco smoke procarcinogens.
\end{abstract}

\section{BACKGROUND AND PURPOSE}

CYP2A enzymes metabolically inactivate nicotine and activate tobacco-derived procarcinogens (e.g. NNK, 4(methylnitrosamino)-1-(3-pyridyl)-1-butanone). Smoking decreases nicotine clearance, and chronic nicotine reduces hepatic CYP2A activity. However, little is known about the impact of smoking or nicotine on the expression of CYP2A in the lung. We investigated 1) the levels of human lung CYP2A mRNA in smokers versus non-smokers and 2) the impact of daily nicotine treatment on lung CYP2A protein levels in African Green Monkeys (AGM).

\section{EXPERIMENTAL APPROACH}

Lung CYP2A13, CYP2A6 and CYP2A7 (and CYP1A2) mRNA levels in smokers and non-smokers were assessed in Gene Expression Omnibus (GEO) datasets (GSE40364, GSE11874 and GSE103845). The impact of chronic twice daily subcutaneous nicotine at two doses $\left(0.3\right.$ and $\left.0.5 \mathrm{mg} \mathrm{kg}^{-1}\right)$, versus vehicle, on lung CYP2A protein levels was assessed. The impact of ethanol self-administration was also investigated, with and without nicotine treatment.

\section{KEY RESULTS}


Smokers, versus non-smokers, had significantly lower levels of lung CYP2A13, CYP2A6 and CYP2A7 (and increased CYP1A2) mRNA in both GEO datasets. Nicotine treatment, at both doses, significantly decreased AGM lung CYP2A protein. Ethanol self-administration had no effect on AGM lung CYP2A protein, and there was no interaction between ethanol and nicotine.

\section{CONCLUSIONS AND IMPLICATIONS}

Smoking was associated with a reduction in human lung CYP2A13, CYP2A6, and CYP2A7 mRNA, consistent with the role of nicotine treatment in reducing AGM lung CYP2A protein. This regulation by smoking/nicotine will increase interindividual variation in lung CYP2A levels that may impact the localized metabolism of inhaled drugs and tobacco smoke procarcinogens.

Keywords

CYP2A13; CYP2A6; African green monkey; nicotine; ethanol; smoking; lung; regulation

\section{Abbreviations}

AGM, African Green Monkey

b.i.d., twice daily

CYP1A2, cytochrome P450 1A2

CYP2A, cytochrome P450 2A isoforms

CYP2A6, cytochrome P450 2A6

CYP2A7, cytochrome P450 2A7

CYP2A13, cytochrome P450 2A13

GEO, Gene Expression Omnibus

NNK, 4-[Methyl(nitroso)amino]-1-(3-pyridinyl)-1-butanone

NNN, 3-(1-Nitrosopyrrolidin-2-yl)-pyridine

s.c., subcutaneous

\section{WHAT IS KNOWN ABOUT THIS SUBJECT}

- CYP2A13 and CYP2A6 are expressed in the lung and may contribute to local procarcinogen activation

- Hepatic CYP2A is downregulated by nicotine and smoking in mice and AGMs

\section{WHAT THIS STUDY ADDS}

- Effect of smoking and nicotine on human and AGM lung CYP2A expression

- Lung CYP2A expression was decreased by systemic treatment with nicotine

\section{WHAT IS THE CLINICAL SIGNIFICANCE}

- Decrease of lung CYP2A expression by nicotine may alter smoking-related lung cancer risk

- Nicotine replacement therapies may have potential benefits on smoking-related lung cancer risk, as well as smoking cessation

\section{Introduction}

The lung is the primary organ exposed to the potentially detrimental effects of inhaled drugs and airborne toxins. These effects include disruption of tissue function through DNA damage by toxic metabolites mediated, at least in part, by cytochrome P450 enzymes (CYPs) expressed in the lung (Godschalk et al., 2002). Regulation of CYPs in the lungs may be important in altering local CYP-mediated metabolism. While 
regulation of CYP expression is well documented for some extrahepatic tissues (Pavek \& Dvorak, 2008), little is known about the regulation of CYPs in the lungs.

Cigarette smoking is a known risk factor for lung cancer (Surgeon \& Health, 2004). CYP2A13 and CYP2A6 are human CYP2A isoforms expressed in adult human liver and lung, that can metabolically activate procarcinogens found in cigarette smoke such as the major tobacco-specific nitrosamines: 4-(methylnitrosamino)1-(3-pyridyl)-z-butanone (NNK) and 3-(1-nitrosopyrrolidin-2-yl)-pyridine (NNN) (Rossini et al., 2008; Su et al., 2000; Zhang et al., 2007). Genetic variation in both CYP2A13and CYP2A6 is associated with altered risk for smoking-derived lung cancer (McKay et al., 2017; Rossini et al., 2008; Wassenaar et al., 2011; Zhang et al., 2007). For example, smokers with $C Y P 2 A 13$ or $C Y P 2 A 6$ genetic variants (e.g. reduced metabolizers) have a lower incidence of lung cancer compared to those without these variants (e.g. normal metabolizers) (D'Agostino et al., 2008; Wassenaar et al., 2015).

CYP2A enzymes are also the primary enzymes responsible for nicotine metabolism in several species including humans (Messina et al., 1997), mice (Murphy et al., 2005), and African green monkeys (AGM) (Schoedel et al., 2003). Cigarette smokers have lower nicotine clearance than non-smokers, thought to be due to down regulation of hepatic CYP2A6 (Zevin \& Benowitz, 1999). In mice pretreated with nicotine for 3 days nicotine metabolism was reduced (Stalhandske \& Slanina, 1970). Similarly, nicotine treatment can downregulate its own metabolism in AGMs. This effect was, at least in part, through reduced hepatic CYP2A mRNA, protein and resulting enzyme activity (Ferguson et al., 2012; Schoedel et al., 2003). As nicotine can decrease hepatic CYP2A mRNA, protein and activity (Ferguson et al., 2012; Schoedel et al., 2003), we hypothesized that smoking, or nicotine exposure, may also reduce lung CYP2A expression. Such xenobiotic-mediated variation in lung CYP2A levels may act in concert with genetic variation in contributing to the risk of smoking-derived damage to lungs.

We used human GEO datasets (O'Beirne et al., 2018; Tilley et al., 2011; Wang et al., 2012) to investigate the association of smoking with human lung CYP2A mRNA levels. We used a non-human primate model (i.e. AGM) to investigate the effect of chronic nicotine treatment on lung CYP2A protein levels. Human alcoholics have higher rates of CYP2A6 activity (Chenoweth et al., 2014), and hepatic CYP2A6 levels than non-alcoholics (Niemela et al., 2000), and alcohol-treated mice have higher hepatic CYP2A than controls (Lu et al., 2011). Therefore, we also conducted an exploratory investigation of the effect of self-administered ethanol, with and without nicotine treatment, on lung CYP2A.

\section{Methods}

Gene expression analyses

RNA-sequence datasets were downloaded from the NCBI Gene Expression Omnibus (GEO) database (https://www.ncbi.nlm.nih.gov/geo/). The datasets were selected based on having data on lung tissue and having sufficient numbers of both healthy smokers and healthy non-smokers. GSE30063 had n=60 smokers and $n=73$ non-smokers (Wang et al., 2012), and GSE108134 had n=129 smokers and $n=29$ non-smokers (O'Beirne et al., 2018). A separate GEO dataset, GSE11784, was used to assess the association of smoking exposure and CYP2A13, CYP2A6 and CYP2A7 mRNA levels. GSE11874 had n=94 smokers with individual data on pack-years of cigarette smoking (Tilley et al., 2011). Pack-years of cigarette smoke were used as a rough estimate of life long exposure to smoking (Leffondre et al., 2002). All three studies examined small airway epithelium of the lungs (O'Beirne et al., 2018; Wang et al., 2012). RNA was hybridized using the Affymetrix Human Genome U133 Plus 2.0 Array (HG-U133_Plus_2) (O'Beirne et al., 2018; Wang et al., 2012).

Raw preprocessed Affymetrix CEL files were downloaded using the Bioconductor GEOquery v2.54.1 Rpackage from GEO (Davis \& Meltzer, 2007). Within each dataset, raw Affymetrix multiarray data from smokers and non-smokers were compiled together using Bioconductor Affy v2.3.01 with corresponding hgu133plus2.db and hgu133plus2cdf R-packages (Carvalho, 2015). Raw readings were processed with the robust multiarray averaging (RMA) pipeline to correct for probe background, to quantile normalize data, to median polish data, and to calculate mRNA probe intensity (Irizarry et al., 2003). The generated 
mRNA probe intensities for each individual were exported into a comma-separated values (.CSV) file (Davis \& Meltzer, 2007). The corresponding gene probe IDs: 208327_at (CYP2A13; NM_000766), 207244_x_at (CYP2A6; NM_000762), 1549_at (CYP2A7; NM_000764), and 207608_X_at (CYP1A2; NM_000761) were used to extract the relative levels of CYP gene probe intensities in individual smokers and non-smokers from the .CSV file.

\section{Animals}

All experimental procedures and animal care were reviewed and approved by the Institutional Review Board of the Behavioural Sciences Foundation and the University of Toronto Animal Care Committee and were conducted in accordance with the guidelines of the Canadian Council of Animal Care. All studies involving animals are reported in accordance with the ARRIVE guidelines for experiments involving animals and with journal recommendations (Kilkenny et al., 2010; McGrath et al., 2010).

Male adult (6-8 years-old, weight range 5-6 kg) African green monkeys (AGM) (Chlorocebus aethiops) were housed outdoors in social groups at the Behavioural Sciences Foundation (St. Kitts) (Palmour et al., 1997). AGMs were obtained from a nonendangered, large and isolated population in the Caribbean. Their diet comprised monkey chow supplemented with fresh fruits and vegetables and drinking waterad libitum .

Animal treatments

\section{AGM study 1: Control and nicotine treatments}

The study paradigm is outlined in Figure 1 and has been previously described in detail (Schoedel et al., 2003). During Phase I (day 0), AGMs ( $\mathrm{n}=12$ ) were randomized by weight into two treatment groups, Control $(\mathrm{n}=6)$ and Nicotine $(\mathrm{n}=6)$. During Phase II (days 1-22), AGMs in the Nicotine group received nicotine bitartrate (mg nicotine base in saline, $\mathrm{pH}$ 7.4) subcutaneous (s.c.) injections twice daily (b.i.d.), at $0.05 \mathrm{mg} \mathrm{kg}^{-1}$ for 2 days, then $0.15 \mathrm{mg} \mathrm{kg}^{-1}$ for 2 days, and then $0.30 \mathrm{mg} \mathrm{kg}^{-1}$ for 18 days. During the last 18 days, the total daily dose of nicotine administered $\left(0.6 \mathrm{mg} \mathrm{kg}^{-1}\right)$ was similar to the average daily intake of nicotine by smokers (0.53 $\mathrm{mg} \mathrm{kg}^{1}$ per day) (Benowitz \& Jacob, 1984). AGMs in the Control group received saline (i.e. vehicle) s.c. injections b.i.d. during this phase.

On day 22, 4 hours after the first s.c. injection of the day, the AGMs were sacrificed under ketamine anaesthesia. AGMs were given a pentobarbital overdose followed by bilateral thoracotomy. Their organs were immediately dissected, and one half of each organ was flash-frozen and stored at $-80^{\circ} \mathrm{C}$ until further use.

AGM study 2: Control, nicotine, ethanol, and nicotine + ethanol treatments

The study paradigm is outlined in Figure 2 and has been previously described in detail (Ferguson et al., 2011). During Phase 1 (days 1-14), AGMs underwent an ethanol preference screen, with daily access to $10 \%$ ethanol in a $0.5 \%$ sucrose solution every morning for 4 hours day ${ }^{-1}$. AGMs $(n=40)$ that consumed more than $1 \mathrm{~g}$ of ethanol $\mathrm{kg}^{-1}$ per day were randomized into four treatment groups based on their ethanol consumption: Control $(n=10)$, Nicotine $(n=10)$, Ethanol $(n=10)$, and Nicotine + Ethanol $(n=10)$. During Phase II (days 15-28), there was a washout period where the AGMs had no drug exposure.

During Phase III (days 29-42), AGMs in the Ethanol and in the Nicotine + Ethanol groups were allowed to self-administer $10 \%$ ethanol in a $0.5 \%$ sucrose solution every morning for 4 hours, while AGMs in the Control and in the Nicotine groups were allowed to self-administer $0.5 \%$ sucrose solution (i.e. vehicle) during the same 4 hour window.

During Phase IV (days 43-64), AGMs continued to have access to $10 \%$ ethanol in a $0.5 \%$ sucrose solution or $0.5 \%$ sucrose solution every morning for 4 hours. In addition, AGMs in the Nicotine, and Nicotine plus Ethanol groups were given nicotine bitartrate ( $\mathrm{mg}$ of nicotine base in saline, $\mathrm{pH}$ 7.0) s.c. injections b.i.d. at $0.05 \mathrm{mg} \mathrm{kg}-1$ for 1 day, then $0.1 \mathrm{mg} \mathrm{kg}{ }^{-1}$ for 1 day, then $0.25 \mathrm{mg} \mathrm{kg}^{-1}$ for 1 day, followed by $0.5 \mathrm{mg} \mathrm{kg}^{-1}$ for the remaining days. AGMs in the Ethanol and Control groups received saline (i.e. vehicle) s.c. injections b.i.d. during this phase. 
On day 64,4 hours after the first s.c. treatment injection, the AGMs were sacrificed under ketamine anaesthesia. AGMs were given a pentobarbital overdose followed by bilateral thoracotomy. Organs were immediately dissected, and one half was frozen and stored at -80degC until further use.

Whole membrane preparation

Lower and upper regions of AGM lungs were dissected by visual differentiation. Whole membranes were prepared from individual AGM lungs as previously described (Miksys \& Tyndale, 2002). Briefly, lung tissues were homogenized in $0.1 \mathrm{mM}$ EDTA, $0.1 \mathrm{mM}$ DDT, $100 \mathrm{mM}$ Tris, and $0.32 \mathrm{M}$ sucrose (pH 7.4), then centrifuged at $3000 \mathrm{x}$ g for 10 minutes at $4 \mathrm{degC}$. The supernatant was collected, the pellet was resuspended, vortexed and centrifuged again at $3000 \mathrm{x}$ g for 10 minutes. The two supernatants were combined and centrifuged at $110,000 \mathrm{x}$ g for 60 minutes at $4 \mathrm{deg}$. The resulting pellet was resuspended in storage solution consisting of $0.1 \mathrm{mM}$ EDTA, $0.1 \mathrm{mM}$ DDT, $100 \mathrm{mM}$ Tris with $1.15 \% \mathrm{KCl}$ and $20 \%$ glycerol. The protein contents of the lung membranes were measured using a Bradford Protein Assay kit (Bio-Rad; Burlington, ON, Canada). Pooled Control group lung membranes for lower and upper lung were created by combining equal amounts of lung membrane protein from each control AGM in Study 1 ( $\mathrm{n}=6)$ and separately for Study $2(\mathrm{n}=10)$.

Immunoblotting analysis

The immunoblotting procedures comply with the recommendations made by the journal.

\section{CYP2A and loading control analyses}

Control lung membrane proteins (ranging from 25-150 $\mu \mathrm{g}$ ) were separated by SDS-PAGE at constant voltages of $60 \mathrm{~V}$ through a $4 \%$ acrylamide stacking gel and of $100 \mathrm{~V}$ through a $10 \%$ acrylamide separating gel at room temperature $\left(22^{\circ} \mathrm{C}\right)$. All immunoblotting procedures were carried out at room temperature unless specified otherwise. A portion of the gel (i.e. ${ }^{\sim} 35 \mathrm{kDa}$ to $\sim 10 \mathrm{kDa}$ ) that did not contain our proteins of interest was stained with EZBlue Gel Coomassie Staining (Sigma; Oakville, ON, Canada) to quantify the protein loaded per well. Proteins in the remaining portion of the gel were transferred to nitrocellulose membrane at a constant current of $300 \mathrm{mAmp}$ for 16 hours. Membranes were then rinsed three times for 5 minutes each in Tris-buffered saline (pH 7.4) with $0.1 \%$ Triton X-100 (TBST).

To detect CYP2A, membranes were blocked for 1 hour with a blocking solution consisting of $3 \%$ skim milk powder, $1 \%$ horse serum, and $0.5 \%$ bovine serum albumin (BSA) in TBST. Membranes were then incubated for 16 hours at $4^{\circ} \mathrm{C}$ with a polyclonal rabbit anti-CYP2A6 antibody (Sigma; Oakville, ON, Canada) diluted 1:1000 in $0.5 \%$ horse serum and $0.5 \%$ BSA in TBST. Membranes were rinsed 3 times for 5 minutes each in TBST, then incubated with fresh blocking solution for 1 hour, then with horseradish peroxidase (HRP)conjugated anti-rabbit secondary antibody (EMD Milipore; Etobicoke, ON, Canada) diluted 1:10000 in 0.5\% horse serum and $0.5 \%$ BSA in TBST for 1 hour.

CYP2A proteins were visualized using an enhanced chemiluminescence (ECL) Immunoblot kit (ThermoFisher; Oakville, ON Canada). CYP2A proteins and Coomassie blue-stained proteins were imaged and quantified with a ChemiDoc MP imaging system equipped with ImageLab v6.0.1 build 34 imaging software. Exposure times of 30 and 60 seconds were used, enabling the detection of CYP2A and Coomassie blue-stained proteins without signal saturation.

After analysis for CYP2A, $\beta$-actin was quantified to further assess protein loading. Membranes were washed for 24 hours in TBST, incubated for 1 hour in fresh blocking solution, and then for 16 hours at $4^{\circ} \mathrm{C}$ with a mouse monoclonal anti- $\beta$-actin antibody (Sigma; Oakville, ON, Canada) diluted 1:1000 in 0.5\% horse serum and $0.5 \%$ BSA in TBST. Membranes were then washed three times for 5 minutes each in TBST, re-blocked for 1 hour, then incubated for 1 hour in HRP-conjugated anti-mouse secondary antibody (EMD Millipore; Etobicoke, ON, Canada) diluted 1:10000 in $0.5 \%$ horse serum and $0.5 \%$ BSA in TBST. $\beta$-actin proteins on blots were imaged and quantified using the ChemiDoc MP imaging system described above using an automatic exposure time to minimize band saturation and maximize signal linearity. 


\section{Antibody specificity and protein linearity}

Pooled Control group lung membranes from the lower and upper lung were serially diluted to generate a standard curve to assess the linear detection range of CYP2A, $\beta$-actin and total proteins (i.e. Coomassie blue-stained protein). Individual AGM samples were loaded at $75 \mu \mathrm{g}$ beside the pooled Control standard curve at $25 \mu \mathrm{g}, 75 \mu \mathrm{g}$, and $150 \mu \mathrm{g}$ on each immunoblot. The standard curve was used to ensure that each membrane sample was in the linear range and to allow for blot-to-blot adjustments. Each blot had a prestained protein mixture in one lane for molecular weight estimation (PaperRuler Prestained Protein Ladder, ThermoFisher; Mississauga, ON, CA).

The specificity and sensitivity of the polyclonal anti-CYP2A6 antibody was assessed using positive controls including human liver microsomes, AGM liver microsomes, AGM lung membranes and membranes from cDNA expressed human CYP2A6 (Corning; Corning NY, USA), and CYP2A13 (XenoTech; Lenexa, KS, USA). Negative controls included cDNA expressed human CYP2E1 (BD Bioscience; Woburn, MA, USA), CYP2D6 (XenoTech; Lenexa, KS, USA), CYP2B6 (BD Bioscience; Woburn, MA, USA), and CYP3A4 (BD Bioscience; Woburn, MA, USA).

Data and statistical analysis

The data and statistical analyses complied with the recommendations of experimental design and analysis in pharmacology (Curtis et al., 2018). The data were analyzed using GraphPad Prism version 7.1a. A p-value of $<0.05$ was considered statistically significant.

\section{GEO multiarray statistical analysis}

Nonparametric unpaired Mann-Whitney U-tests were used to test differences in CYP2A13, CYP2A6 and CYP2A7 expression levels between smokers and non-smokers in the small airway epithelium of the lung in GEO datasets: GSE30063 and GSE108134.

For GSE11874, the D'Agostino-Pearson omnibus normality test was used to determine the normality of signal distribution of CYP2A13, CYP2A6 and CYP2A7 expression levels in smokers' small airway epithelium of the lung, followed by correlation analyses to assess the relationship between CYP2A13, CYP2A6 and CYP2A7 expression levels and pack-year data, within smokers.

\section{Background subtraction and blot-to-blot adjustment of immunoblot data}

Individual AGM samples were assessed on multiple immunoblots.For all signals (CYP2A, $\beta$-actin, and Coomassie blue-stain), software automatic background subtraction was applied to all blots. CYP2A signals were then normalized to $\beta$-actin signals or Coomassie blue-stained total protein to adjust for variation in protein loading (see Supplementary Tables 1 and 2). Further background subtraction was applied, after this normalization to loading controls, based on the y-intercept from the linear regression analysis of the CYP2A/ $\beta$-actin or CYP2A/Coomassie control group pooled standard curves present on each immunoblot. To reduce blot-to-blot variation in the analyses, the individual AGM signals were adjusted using the $75 \mu \mathrm{g}$ of pooled Control group lung membrane signal (internal standard). Analyses with (illustrated in the figures), and without, this additional blot-to-blot adjustment had no impact on the fold or statistical significance of differences (Supplementary Tables 1 and 2).

\section{Immunoblot statistical analysis}

Two-tailed unpaired t-tests were used to assess differences in the lung CYP2A protein levels between treatment groups in AGM study 1. Two-way ANOVA tests were used to assess differences in the lung CYP2A protein levels between treatment groups in AGM study 2. Tukey's multiple comparison post hoc tests were subsequently used to assess differences in lung CYP2A protein levels between treatment groups and treatment interactions in AGM study 2. The D'Agostino-Pearson omnibus normality test was used to determine the normality of signal distribution, followed by correlation analyses to assess the relationships between lower and upper lung CYP2A protein levels within individual AGMs. Data from both AGM studies were found to 
be normally distributed; thus, Pearson's correlations were used to assess the relationship between the lower and upper lung.

\section{AGM Liver comparisons}

The CYP2A levels in AGM livers from the AGM study 2 Control group have been previously quantified (Ferguson et al., 2012). Using these data, the total liver and total lung CYP2A were estimated and compared using a paired two-tailed t-test.

Materials

Human liver microsomes were prepared in a previous study (Al Koudsi et al., 2010). Microsomes were prepared as a pool of 17 livers from the Biocentre in Basel, Switzerland (23). AGM liver microsomes were prepared in a previous study (Ferguson et al., 2011). Microsomes were prepared as a pool of 10 livers from the Control group AGM from Study 2.

Human CYP2A13 (Cat \#: EZ033) and CYP2D6 (Cat \#: EZ013) bactosomes were purchased from SEKISUI XenoTech (KS, USA). Human 2A6 enzyme microsomes (Cat \#: 456204) were purchased from CORNING Inc. (NY, USA). Human CYP2B6 (Cat \#: 456255), CYP3A4 (Cat \#: 455307) and CYP2E1 (Cat \#: 456255) enzyme microsomes were purchased from BD Bioscience Company (MA, USA). All other reagents were obtained from standard suppliers. Information about the antibodies used can be found in Supplementary Table 3 .

\section{Results}

CYP2A13, CYP2A6, and CYP2A7 mRNA were lower in smokers than in non-smokers

Smokers, vs non-smokers, had significantly lower levels of CYP2A13, CYP2A6 and CYP2A7 mRNA in the small airway epithelium of the lung; this finding was replicated in two GEO datasets (Figure 3). Smoking is an established inducer of CYP1A2 mRNA and thus was used as a positive control for the assessment within this data set of the association of smoking status and viability of samples (Wei et al., 2002). Smokers, vs non-smokers, had significantly higher CYP1A2 mRNA in both datasets.

In GSE11784, smoking quantity was assessed, using pack-year data, against levels of CYP2A13, CYP2A6 and CYP2A7 mRNA in the small airway epithelium of the lung. There was a significant, albeit weak, negative correlation between CYP2A13 ( $\mathrm{r}=-0.178)$, CYP2A6 $(\mathrm{r}=-0.307)$ and, CYP2A7 $(\mathrm{r}=-0.198)$ mRNAs and pack-years smoked, suggesting a potential dose-dependent association between smoking exposure and CYP2A mRNA levels in smokers' lungs.

CYP2A protein levels were detected in AGM lung by immunoblotting

An immunoblotting assay was established using a polyclonal anti-CYP2A6 antibody to measure the levels of CYP2A protein in AGM lung. A CYP2A immunoreactive signal was detected in human liver, AGM liver, AGM lung, and cDNA-expressed CYP2A13 and CYP2A6 (Figure 4A), while there was no cross-reactivity with other cDNA-expressed human CYPs (CYP3A4, CYP2E1, CYP2D6, and CYP2B6) (Figure 4A). The assays were linear for detection of CYP2A, $\beta$-actin and Coomassie blue signals over a wide range of AGM lung protein (Figure 4B-D).

Nicotine treatment lowered CYP2A protein levels in the lower and upper lung in AGM study 1

Most AGM lung samples, loaded at $75 \mu \mathrm{g}$, were within the linear range of the CYP2A, $\beta$-actin and Coomassie blue signals from the standard curve of pooled control AGM lung (described in the Methods) on each immunoblot (Figure 5A and 5B). However, a few samples had CYP2A detection which were below the linear detection level and were thus reassessed after loading at $125 \mu \mathrm{g}$. A representative blot from AGM study 1 is shown in Figure 5A and 5B; representative AGM lung membranes from each group $(n=3)$ were loaded at $75 \mu \mathrm{g}$. 
The Nicotine group, vs the Control group, had significantly lower levels of CYP2A protein in the lower (4.03-fold) and upper (3.74-fold) lung (Figure 5C and 5D). Blot-to-blot adjusted (as shown in Figure 5C-D) and unadjusted data provided virtually identical significant fold group differences (Supplementary Table 1).

CYP2A protein levels in the lower and upper lung were highly correlated $\left(\mathrm{r}^{2}=0.88\right)$, within individual AGMs (Figure $5 \mathrm{E}$ ). There were no significant differences in CYP2A $/ \beta$-actin levels between the lower and upper lung of the Control group or Nicotine group.

Nicotine, but not ethanol treatment, lowered CYP2A protein levels in the lower and upper lung in AGM study 2

For many samples in the Nicotine and Nicotine + Ethanol groups, the CYP2A detection was below the linear detection level when loaded at $75 \mu \mathrm{g}$. Thus, samples from both of these groups were assessed at $100 \mu \mathrm{g}$ of loaded protein. Most AGM lung samples in the Control and Ethanol groups (loaded at $75 \mu \mathrm{g}$ ) and Nicotine and Nicotine + Ethanol group (loaded at $100 \mu \mathrm{g}$ ) were within the linear range of the CYP2A, $\beta$-actin and Coomassie blue signals from the standard curve of pooled control AGM lung (described in Methods) on each immunoblot. However, a few samples had CYP2A detection which was above, or below, the linear detection and were thus reassessed after loading at $25 \mu \mathrm{g}$ or $125 \mu \mathrm{g}$, respectively. A representative blot from AGM study 2 is shown in Figure $6 \mathrm{~A}$ and $6 \mathrm{~B}$, representative AGM lung membranes from each group ( $\mathrm{n}=3$ ) were loaded at $75 \mu \mathrm{g}$.

There was a significant main effect of the nicotine vs control treatments in both the lower and upper lung. There was no main effect of self-administration of ethanol vs control treatments in either the lower or upper lung. There was also no treatment interaction (Figure $6 \mathrm{C}$ and $\mathrm{D}$ ).

The Nicotine group, vs the Control group, had significantly lower levels of CYP2A protein in the lower (5.87-fold) and upper (4.46-fold) lung (Figure 6C and D). The Nicotine + Ethanol group, vs the Control group, also had significantly lower levels of CYP2A protein in the lower (6.83-fold) and upper (6.73-fold) lung. However, the Nicotine group, vs the Nicotine + Ethanol group, did not have significantly different levels of CYP2A protein in the lower or upper lung. Blot-to-blot adjusted (as shown in Figure 6C-E), and unadjusted (raw) data, provided virtually identical significant fold group differences (Supplementary Table 2).

CYP2A protein levels, in the lower and upper lung, were highly correlated $\left(\mathrm{r}^{2}=0.78\right)$, within individual AGMs (Figure 6E). There were no significant differences in CYP2A $/ \beta$-actin levels between the lower and upper lung of the Control group, Nicotine group, Ethanol group, or Nicotine + Ethanol group.

Within AGMs, CYP2A protein levels were lower in the lung compared to the liver

Hepatic CYP2A protein levels from individual AGMs in AGM study 2 have been previously analyzed by our group (Supplementary Table 3)(Ferguson et al., 2012). The lung, vs the liver, had significantly and substantially (approximately 40-fold) lower levels of CYP2A protein (Supplementary Figure 1), as expected. CYP2A protein levels, in the liver and lung were also highly correlated within individual AGMs (Supplementary Figure 1).

\section{Discussion}

The findings of this study indicate that smoking and nicotine exposure can reduce CYP2A enzymes in human and AGM lung. In humans, CYP2A isoforms of CYP2A13, CYP2A6 and CYP2A7 mRNAs were lower in the lungs of smokers compared to non-smokers, which was observed in two GEO datasets (O'Beirne et al., 2018; Wang et al., 2012). Consistent with this, in two non-human primate studies, AGMs treated with nicotine had lower levels of lung CYP2A protein than controls. The $0.3 \mathrm{mg} \mathrm{kg}^{-1}$ nicotine treatment in study 1 resulted in a 4.03-fold and 3.74-fold decrease in CYP2A protein for the lower and upper lung, and the $0.5 \mathrm{mg} \mathrm{kg}^{-1}$ nicotine treatment in study 2 resulted in a 5.87 - and 4.46 -fold decrease in the lower and upper lung, respectively. The apparent greater fold-decrease in AGM study 2 compared to study 1 suggests a potential dose-related impact of nicotine treatment on lowering AGM lung CYP2A. This was also reflected 
in the human data where CYP2A mRNA was negatively correlated with cigarette pack-years. The effect of nicotine on lung CYP2A was similar throughout the lung with no significant difference in impact between the lower and upper regions. Ethanol exposure did not affect AGM lung CYP2A, alone or in combination with nicotine, suggesting a lack of effect and synergism between these two drugs under these conditions. Within AGM study 2, we also highlighted the relative expression of CYP2A in the lung compared to the liver: 40-fold lower level of total AGM CYP2A protein in the lung than in the liver (Supplementary Figure 1). Human CYP2A mRNA levels in various respiratory tissues were also lower compared to the liver : 14 -fold lower in the nasal mucosa, 67 -fold lower in the trachea, and 144-fold lower in the lungs (Su et al., 2000). The similarity in relative organ levels of CYP2A suggests that for liver and lung there is similar control over expression of CYP2As in AGMs and humans.

Previous reports on CYP2A detection and quantification in human lung have been inconsistent. In a study on cancer patients, the levels of CYP2A13 and CYP2A6 proteins from peripheral lung surgical resections (adjacent to the tumor) varied extensively between patients (Zhang et al., 2007). Some patients had low levels of lung CYP2A protein; $\sim 10 \%$ had no detectable CYP2A6 and $~ 88 \%$ had no detectable CYP2A13 (Zhang et al., 2007). In the current study, CYP2A6 and CYP2A13 mRNAs were detectable in all subjects. Differences between healthy and resected tissues may have contributed to this difference in detection between the current and previous studies. In mice, lung tumors were found to drastically downregulate the expression of CYP2A in adjacent tissue, approximately ${ }^{\sim}$ 2-fold decrease in CYP2A13 protein and mRNA compared to control healthy lung tissue (Liu et al., 2015); this may partially explain the lack of CYP2A detection in the previous human study (Zhang et al., 2007). The current study also highlights an underlying effect of smoking/nicotine in lowering lung CYP2A expression. Thus, we suspect that patient exposure to smoking or nicotine may also have contributed to the low CYP2A protein detection in the previous study (Zhang et al., 2007).

There was a lower fold-difference in human lung CYP2A mRNA by smoking status (1.04-1.12-fold) compared to the fold-difference in AGM lung CYP2A protein by nicotine treatments $\left({ }^{\sim} 3-7\right.$-fold $)$. This may be due to transcriptional/translational differences and/or species differences. However, AGM CYP2A is expected to have high homology and overlapping regulation with human CYP2A13 and CYP2A6. While AGM CYP2A has not yet been sequenced, CYP2A24 of Cynomolgus monkeys (another old-world monkey) has 94\% and 93\% nucleotide identity with human CYP2A13 and CYP2A6, respectively (Shimada et al., 2016). Another plausible explanation relates to nicotine exposure differences between human smokers and nicotine-treated AGMs. The average plasma nicotine levels of smokers is $30 \mathrm{ng} \mathrm{ml}^{-1}$, which is similar to the average plasma nicotine levels of $29.5 \mathrm{ng} \mathrm{ml}^{-1}$ achieved in AGM study 1 (Lee et al., 2006), and $40.7 \mathrm{ng} \mathrm{ml}^{-1}$ achieved in AGM study 2 (Ferguson et al., 2011). However, nicotine was administered to AGMs in two bolus s.c. injections, compared to nicotine obtained from 20 cigarettes smoked throughout the span of a day by the average smoker (Benowitz \& Jacob, 1984). The resulting higher peak concentrations of plasma nicotine in AGMs compared to the average smoker, may have impacted the nicotine effect in AGM compared to human lung CYP2A. There are also differences in the specific xenobiotic exposure, where tobacco smoke may contain potential lung CYP2A up-regulators, as well as down-regulators, such as nicotine.

Ethanol self-administration had no apparent effect on AGM lung CYP2A levels. The ethanol treatment paradigm was designed to model moderate alcohol consumption. The average daily ethanol intake of monkeys in the Ethanol and Nicotine+Ethanol groups was approximately $3.0 \mathrm{~g}$ of ethanol per $\mathrm{kg}$ of body weight (Ferguson et al., 2011). This level of consumption in these monkeys led to an average \pm S.D. blood ethanol level of $26 \pm 3.0 \mathrm{mM}$ (Ferguson et al., 2011), which is within the range found in moderate alcohol consumers, 5-20 mM (Eckardt et al., 1998). Ethanol has been shown to increase CYP2A6 in human monocytic cell lines, where ethanol treatment at $100 \mathrm{mM}$ was able to induce CYP2A6 (Jin et al., 2012). Additionally, the nicotine metabolite ratio (NMR), an in vivopredictive CYP2A6 biomarker, was positively correlated with drinks per week (Chenoweth et al., 2014) and those in treatment for alcohol-dependence had a decrease in NMR with abstinence, suggesting heavy alcohol-use may induce hepatic CYP2A6 (Dermody et al., 2020). Thus, it is possible that higher ethanol consumption than found here may induce lung CYP2A. 
In the current study, the effects of nicotine and ethanol exposure on AGM lung CYP2A were consistent with our previous findings in these AGMs, where AGM hepatic CYP2A was significantly lowered by nicotine treatment, and unaffected by ethanol (Ferguson et al., 2012). There was an apparent larger effect of nicotine treatment on CYP2A expression in lung than in liver. In AGM study 1 nicotine treatment $(0.3 \mathrm{mg}$ $\mathrm{kg}^{-1}$ nicotine), decreased hepatic CYP2A by $\sim 1.6$-fold (Schoedel et al., 2003), less than the $\sim 3.8$-fold-difference observed here in lung CYP2A. Similarly, in AGM study 2 nicotine treatment $\left(0.5 \mathrm{mg} \mathrm{kg}^{-1}\right.$ nicotine), decreased hepatic CYP2A by $\sim 2.5$-fold (Ferguson et al., 2012) versus the $\sim 5.2$-fold-difference observed here in lung CYP2A. Together this suggests that these nicotine treatments may cause a larger reduction in CYP2A expression in AGM lung than liver. Furthermore, the association of smoking in humans, and the systemic nicotine treatment of AGMs (s.c. injections), with reduced lung CYP2A suggest that nicotine itself is capable of reducing CYP2A in the lungs and liver even after a non-pulmonary delivery. Thus, we suspect that nicotine delivered by other routes, such as by nicotine patch, may also down-regulate CYP2A.

There are many potential consequences of lowered CYP2A expression in the lung and the liver. In humans, both CYP2A13 and CYP2A6 can metabolically activate a range of procarcinogens, including NNN and NNK, found in cigarette smoke (D'Agostino et al., 2008; McKay et al., 2017; Wassenaar et al., 2015). For NNK, lung CYP-mediated activation may play a larger role in lung carcinogenicity than hepatic activation (Li et al., 2014; Liu et al., 2015; Weng et al., 2007; Zhang et al., 2007). NNK induced fewer lung tumors in an inactivated-lung CYP mice model compared to inactivated-liver CYP mice (Weng et al., 2007), suggesting the lung CYPs contribute more than the hepatic CYPs to NNK-derived tumour formation. (Weng et al., 2007). Hepatic CYP activity, including CYP2A, is still important in smoking-related lung cancer, in part via activation of other procarcinogens, and the relative contributions of lung and hepatic CYP2A may be carcinogen-dependent (Gu et al., 2005).

$C Y P 2 A 13$ and $C Y$ P2A6 genetically reduced-metabolizers are at lower risk for smoking-related lung cancer, which may be due to the reduced activation of smoking-related procarcinogens (D'Agostino et al., 2008; McKay et al., 2017; Wassenaar et al., 2015). CYP2A13reduced-metabolizers with the CYP2A13 *2 allele, a genetic variant with reduced activity toward nitrosamines, were found to be at lower risk for smoking-related adenocarcinoma compared to normalCYP2A13 metabolizers (D'Agostino et al., 2008). Similarly, CYP2A6 reduced-metabolizers have a lower risk for lung cancer compared to normal-metabolizers (Wassenaar et al., 2015). Thus, the effect of nicotine treatment on the reduction of lung CYP2As and previously reported reduction in hepatic CYP2As, suggests that nicotine via pulmonary or systemic delivery may reduce lung cancer risk through the reduction of procarcinogen activation by lung and hepatic CYP2As.

In summary, this is the first study to show the association of smoking with a reduction in human lung CYP2A mRNA. This was supported by the assessment in non-human primates of the causal impact of nicotine treatment on decreasing AGM lung CYP2A protein. The decrease in lung CYP2A by nicotine was similar throughout the lung and was not dependent on pulmonary intake (i.e. occurred with systemic administration). It remains to be determined whether this down-regulation of lung CYP2A by nicotine would be sufficient to alter the risk of lung damage or disease from inhaled or systemic drugs, carcinogens, and toxins.

\section{References}

Al Koudsi, N., Hoffmann, E. B., Assadzadeh, A., \& Tyndale, R. F. (2010). Hepatic CYP2A6 levels and nicotine metabolism: impact of genetic, physiological, environmental, and epigenetic factors. Eur J Clin Pharmacol, 66 (3), 239-251.

Benowitz, N. L., \& Jacob, P., 3rd. (1984). Daily intake of nicotine during cigarette smoking. Clinical pharmacology and therapeutics, 35 (4), 499-504.

Carvalho, B. (2015). pd.hg.u133.plus.2. In

Chenoweth, M. J., Novalen, M., Hawk, L. W., Schnoll, R. A., George, T. P., Cinciripini, P. M., et al. (2014). Known and novel sources of variability in the nicotine metabolite ratio in a large sample of treatment-seeking 
smokers. Cancer Epidemiol Biomarkers Prev, 23 (9), 1773-1782.

Curtis, M. J., Alexander, S., Cirino, G., Docherty, J. R., George, C. H., Giembycz, et al. (2018). Experimental design and analysis and their reporting II: updated and simplified guidance for authors and peer reviewers. Br J Pharmacol, 175 (7), 987-993.

D'Agostino, J., Zhang, X., Wu, H., Ling, G., Wang, S., Zhang, Q.-Y.,et al. (2008). Characterization of CYP2A13*2, a variant cytochrome P450 allele previously found to be associated with decreased incidences of lung adenocarcinoma in smokers. Drug metabolism and disposition: the biological fate of chemicals, 36 (11), 2316-2323.

Davis, S., \& Meltzer, P. S. (2007). GEOquery: a bridge between the Gene Expression Omnibus (GEO) and BioConductor. Bioinformatics (Oxford, England), 23 (14), 1846-1847.

Dermody, S. S., Hendershot, C. S., Andrade, A. K., Novalen, M., \& Tyndale, R. F. (2020). Changes in Nicotine Metabolite Ratio Among Daily Smokers Receiving Treatment for Alcohol Use Disorder. Nicotine Tob Res, 22 (2), 256-263.

Eckardt, M. J., File, S. E., Gessa, G. L., Grant, K. A., Guerri, C., Hoffman, P. L., et al. (1998). Effects of moderate alcohol consumption on the central nervous system. Alcohol Clin Exp Res, 22 (5), 998-1040.

Ferguson, C. S., Miksys, S., Palmour, R., \& Tyndale, R. F. (2011). Independent and combined effects of ethanol self-administration and nicotine treatment on hepatic CYP2E1 in African green monkeys.Drug metabolism and disposition: the biological fate of chemicals, 39 (12), 2233-2241.

Ferguson, C. S., Miksys, S., Palmour, R. M., \& Tyndale, R. F. (2012). Differential effects of nicotine treatment and ethanol self-administration on CYP2A6, CYP2B6 and nicotine pharmacokinetics in African green monkeys. J Pharmacol Exp Ther, 343 (3), 628-637.

Godschalk, R., Nair, J., van Schooten, F. J., Risch, A., Drings, P., Kayser, K., et al. (2002). Comparison of multiple DNA adduct types in tumor adjacent human lung tissue: effect of cigarette smoking. Carcinogenesis, 23 (12), 2081-2086.

Gu, J., Cui, H., Behr, M., Zhang, L., Zhang, Q. Y., Yang, W., et al. (2005). In vivo mechanisms of tissueselective drug toxicity: effects of liver-specific knockout of the NADPH-cytochrome P450 reductase gene on acetaminophen toxicity in kidney, lung, and nasal mucosa. Mol Pharmacol, 67 (3), 623-630.

Irizarry, R. A., Hobbs, B., Collin, F., Beazer-Barclay, Y. D., Antonellis, K. J., Scherf, U., et al. (2003). Exploration, normalization, and summaries of high density oligonucleotide array probe level data. Biostatistics (Oxford, England), 4 (2), 249-264.

Kilkenny, C., Browne, W. J., Cuthill, I. C., Emerson, M., \& Altman, D. G. (2010). Improving bioscience research reporting: The ARRIVE guidelines for reporting animal research. Journal of pharmacology $\& 3$ pharmacotherapeutics, 1 (2), 94-99.

Lee, A. M., Joshi, M., Yue, J., \& Tyndale, R. F. (2006). Phenobarbital induces monkey brain CYP2E1 protein but not hepatic CYP2E1, in vitro or in vivo chlorzoxazone metabolism. European journal of pharmacology, 552 (1-3), 151-158.

Leffondre, K., Abrahamowicz, M., Siemiatycki, J., \& Rachet, B. (2002). Modeling smoking history: a comparison of different approaches. Am J Epidemiol, 156 (9), 813-823.

Li, L., Megaraj, V., Wei, Y., \& Ding, X. (2014). Identification of cytochrome P450 enzymes critical for lung tumorigenesis by the tobacco-specific carcinogen 4-(methylnitrosamino)-1-(3-pyridyl)-1-butanone (NNK): insights from a novel Cyp2abfgs-null mouse. Carcinogenesis, 35 (11), 2584-2591.

Liu, Z., Megaraj, V., Li, L., Sell, S., Hu, J., \& Ding, X. (2015). Suppression of pulmonary CYP2A13 expression by carcinogen-induced lung tumorigenesis in a CYP2A13-humanized mouse model. Drug Metab Dispos, 43 (5), 698-702. 
Lu, Y., Zhuge, J., Wu, D., \& Cederbaum, A. I. (2011). Ethanol induction of CYP2A5: permissive role for CYP2E1. Drug Metab Dispos, 39 (2), 330-336.

McGrath JC, Drummond GB, McLachlan EM, Kilkenny C, \& Wainwright CL (2010). Guidelines for reporting experiments involving animals: the ARRIVE guidelines, pp 1573-1576.

McKay, J. D., Hung, R. J., Han, Y., Zong, X., Carreras-Torres, R., Christiani, D. C., et al. (2017). Large-scale association analysis identifies new lung cancer susceptibility loci and heterogeneity in genetic susceptibility across histological subtypes. Nat Genet, 49 (7), 1126-1132.

Messina, E. S., Tyndale, R. F., \& Sellers, E. M. (1997). A major role for CYP2A6 in nicotine C-oxidation by human liver microsomes. The Journal of pharmacology and experimental therapeutics, 282 (3), 1608-1614.

Miksys, S. L., \& Tyndale, R. F. (2002). Drug-metabolizing cytochrome P450s in the brain. J Psychiatry Neurosci, 27 (6), 406-415.

Murphy, S. E., Raulinaitis, V., \& Brown, K. M. (2005). Nicotine 5'-oxidation and methyl oxidation by P450 2A enzymes. Drug Metab Dispos, 33 (8), 1166-1173.

Niemela, O., Parkkila, S., Juvonen, R. O., Viitala, K., Gelboin, H. V., \& Pasanen, M. (2000). Cytochromes $\mathrm{P} 450$ 2A6, 2E1, and 3A and production of protein-aldehyde adducts in the liver of patients with alcoholic and non-alcoholic liver diseases. Journal of hepatology, 33 (6), 893-901.

O'Beirne, S. L., Shenoy, S. A., Salit, J., Strulovici-Barel, Y., Kaner, R. J., Visvanathan, et al. (2018). Ambient Pollution-related Reprogramming of the Human Small Airway Epithelial Transcriptome.American journal of respiratory and critical care medicine, 198 (11), 1413-1422.

Palmour, R. M., Mulligan, J., Howbert, J. J., \& Ervin, F. (1997). Of monkeys and men: vervets and the genetics of human-like behaviors. American journal of human genetics, 61 (3), 481-488.

Pavek, P., \& Dvorak, Z. (2008). Xenobiotic-induced transcriptional regulation of xenobiotic metabolizing enzymes of the cytochrome P450 superfamily in human extrahepatic tissues. Curr Drug Metab, 9 (2), 129143.

Rossini, A., de Almeida Simao, T., Albano, R. M., \& Pinto, L. F. (2008). CYP2A6 polymorphisms and risk for tobacco-related cancers. Pharmacogenomics, 9 (11), 1737-1752.

Schoedel, K. A., Sellers, E. M., Palmour, R., \& Tyndale, R. F. (2003). Down-regulation of hepatic nicotine metabolism and a CYP2A6-like enzyme in African green monkeys after long-term nicotine administration. Molecular pharmacology, 63 (1), 96-104.

Shimada, T., Kakimoto, K., Takenaka, S., Koga, N., Uehara, S., Murayama, N., et al. (2016). Roles of Human CYP2A6 and Monkey CYP2A24 and 2A26 Cytochrome P450 Enzymes in the Oxidation of 2,5,2',5'Tetrachlorobiphenyl. Drug Metab Dispos, 44 (12), 1899-1909.

Stalhandske, T., \& Slanina, P. (1970). Effect of nicotine treatment on the metabolism of nicotine in the mouse liver in vitro. Acta pharmacologica et toxicologica, 28 (1), 75-80.

Su, T., Bao, Z., Zhang, Q. Y., Smith, T. J., Hong, J. Y., \& Ding, X. (2000). Human cytochrome P450 CYP2A13: predominant expression in the respiratory tract and its high efficiency metabolic activation of a tobacco-specific carcinogen, 4-(methylnitrosamino)-1-(3-pyridyl)-1-butanone. Cancer research, 60 (18), 5074-5079.

Surgeon, O. o. t., \& Health, O. o. S. a. (2004). Reports of the Surgeon General. In The Health Consequences of Smoking: A Report of the Surgeon General . Centers for Disease Control and Prevention (US).

Tilley, A. E., O'Connor, T. P., Hackett, N. R., Strulovici-Barel, Y., Salit, J., Amoroso, N., et al. (2011). Biologic phenotyping of the human small airway epithelial response to cigarette smoking.PLoS One, 6 (7), e22798. 
Wang, R., Ahmed, J., Wang, G., Hassan, I., Strulovici-Barel, Y., Salit, J., et al. (2012). Airway epithelial expression of TLR5 is downregulated in healthy smokers and smokers with chronic obstructive pulmonary disease. J Immunol, 189 (5), 2217-2225.

Wassenaar, C. A., Dong, Q., Wei, Q., Amos, C. I., Spitz, M. R., \& Tyndale, R. F. (2011). Relationship between CYP2A6 and CHRNA5-CHRNA3-CHRNB4 variation and smoking behaviors and lung cancer risk. J Natl Cancer Inst, 103 (17), 1342-1346.

Wassenaar, C. A., Ye, Y., Cai, Q., Aldrich, M. C., Knight, J., Spitz, M. R., et al. (2015). CYP2A6 reduced activity gene variants confer reduction in lung cancer risk in African American smokers-findings from two independent populations. Carcinogenesis, 36 (1), 99-103.

Wei, C., Caccavale, R. J., Weyand, E. H., Chen, S., \& Iba, M. M. (2002). Induction of CYP1A1 and CYP1A2 expressions by prototypic and atypical inducers in the human lung. Cancer Lett, 178 (1), 25-36.

Weng, Y., Fang, C., Turesky, R. J., Behr, M., Kaminsky, L. S., \& Ding, X. (2007). Determination of the role of target tissue metabolism in lung carcinogenesis using conditional cytochrome P450 reductase-null mice. Cancer Res, 67 (16), 7825-7832.

Zevin, S., \& Benowitz, N. L. (1999). Drug interactions with tobacco smoking. An update. Clinical pharmacokinetics, 36 (6), 425-438.

Zhang, X., D'Agostino, J., Wu, H., Zhang, Q.-Y., von Weymarn, L., Murphy, S. E., et al . (2007). CYP2A13: variable expression and role in human lung microsomal metabolic activation of the tobacco-specific carcinogen 4-(methylnitrosamino)-1-(3-pyridyl)-1-butanone. The Journal of pharmacology and experimental therapeutics, 323 (2), 570-578.

Figure Legends

Figure 1. Treatment paradigm for the AGM study 1.

Figure 2. Treatment paradigm for the AGM study 2.

Figure 3. Lung CYP2A13, CYP2A6 and CYP2A7 mRNA levels were lower in smokers than non-smokers. Probe intensities for the three mRNAs in (A) the GSE30063 (GEO Study 1) and (B) the GSE108134 (GEO Study 2) were significantly lower in smokers than in non-smokers (Unpaired Mann-Whitney U tests). * $\mathrm{p}<0.05$.

Figure 4. Immunoblotting assays were selective and linear. (A) Positive controls for CYP2A6 primary antibody included human liver microsomes, AGM liver microsomes and cDNA-expressed CYP2A13 and CYP2A6. Negative controls included cDNA-expressed CYP3A4, CYP2E1, CYP2D6, and CYP2B6; there was no crossreactivity of the CYP2A6 primary antibody with these CYPs. Standard curves of pooled AGM Control group lung membranes display linear signal detection (y-axis units are arbitrary optical densities) for up to 200 $\mu \mathrm{g}$ protein loaded with (B) CYP2A6 antibody, (C) $\beta$-actin antibody and (D) Coomassie blue gel staining (Linear regression analyses demonstrated significant values of $\mathrm{R}^{2}$ ).

Figure 5. AGM Lung CYP2A protein levels were lower in the Nicotine group compared to the Control group (AGM study 1). Lung CYP2A immunoblots from (A) the lower lung and (B) the upper lung are shown with $\beta$-actin immunoblots and Coomassie blue gel staining (protein loading controls). The blots contain a standard curve of pooled control AGM lung $(25-150 \mu \mathrm{g})$, and representative samples loaded at $75 \mu \mathrm{g}(\mathrm{n}=3$ of 6 from each treatment group). CYP2A/ $\beta$-actin was significantly lower in (C) the lower lungs and (D) the upper lungs of the Nicotine group compared to Control group $(n=6$ per group, two-tailed unpaired $t$ test with Welch's correction). E) CYP2A/ $\beta$-actin levels were significantly correlated between the lower and upper lung within AGM (Pearson's r correlation analysis). ${ }^{*} \mathrm{p}<0.05$.

Figure 6. AGM Lung CYP2A protein levels were lower in the Nicotine groups compared to the Control groups with little apparent impact in the Ethanol group (AGM study 2). Lung CYP2A immunoblots from (A) the lower lung and (B) the upper lung are shown with $\beta$-actin immunoblots and Coomassie blue gel 
staining (protein loading controls). The blots contain representative samples loaded at $75 \mu \mathrm{g}(\mathrm{n}=3$ of 10 from each treatment group). CYP2A/ $\beta$-actin was significantly lower in (C) the lower lungs and (D) the upper lungs of Nicotine and Nicotine + Ethanol groups compared to the Control group ( $\mathrm{n}=10$ per group, two-way ANOVA followed by Tukey's multiple comparison post hoc tests). E) CYP2A/ $\beta$-actin levels were significantly correlated between the lower and upper lung within AGM (Pearson's r correlation analysis). * $\mathrm{p}<0.05$.

\begin{tabular}{|c|c|c|c|}
\hline $\begin{array}{l}\text { Days } \\
0\end{array}$ & $\begin{array}{l}\text { Phase I } \\
\text { - AGMs ( } n=12) \text { were randomized into } 2 \text { groups by body } \\
\quad \text { weight }(n=6 / \text { group })\end{array}$ & $\begin{array}{l}\text { Treat } \\
\text { Control } \\
\text { Group }\end{array}$ & $\begin{array}{l}\text { tment } \\
\text { Nicotine } \\
\text { Group }\end{array}$ \\
\hline $1-22$ & $\begin{array}{l}\text { Phase II } \\
\text { 1. Control: Saline (vehicle) s.c. injections twice daily (b.i.d.) } \\
\text { 2. Nicotine: Nicotine (in saline) s.c. injections b.i.d. at: } \\
\text { Day 1-2: } 0.05 \mathrm{mg} \mathrm{kg}^{-1} \\
\text { Day 3-4: } 0.15 \mathrm{mg} \mathrm{kg}^{-1} \\
\text { Day 5-22: } 0.30 \mathrm{mg} \mathrm{kg}^{-1}\end{array}$ & & \\
\hline & $\begin{array}{l}\text { Day 22: } 4 \text { hours following first s.c. injection treatment, AGMs in } \\
\text { both groups were sacrificed }\end{array}$ & & \\
\hline
\end{tabular}




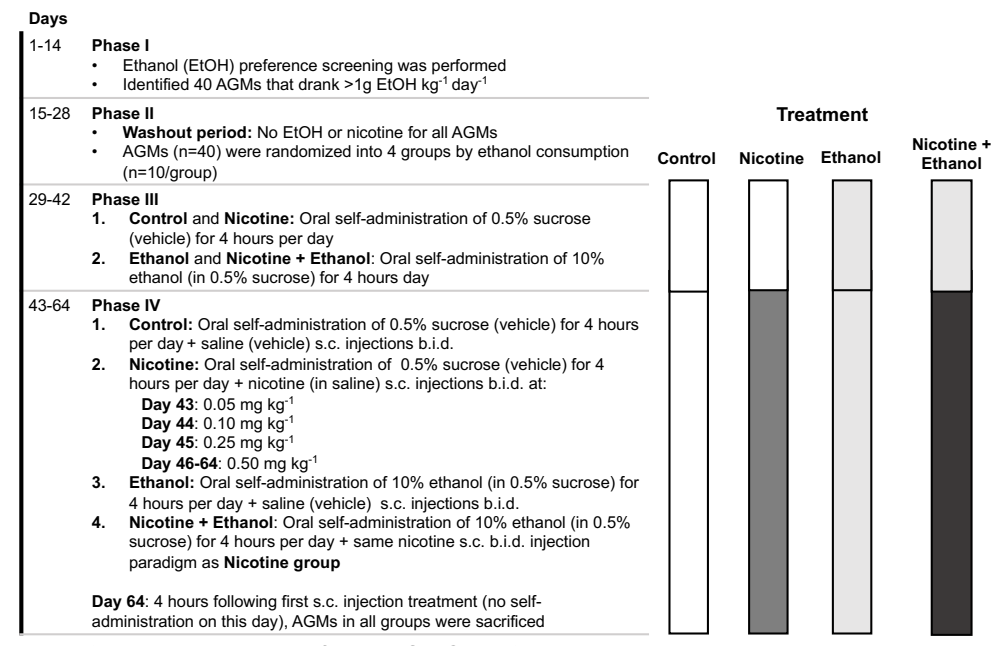

Figure 2. Treatment paradigm for the AGM Study 2. 

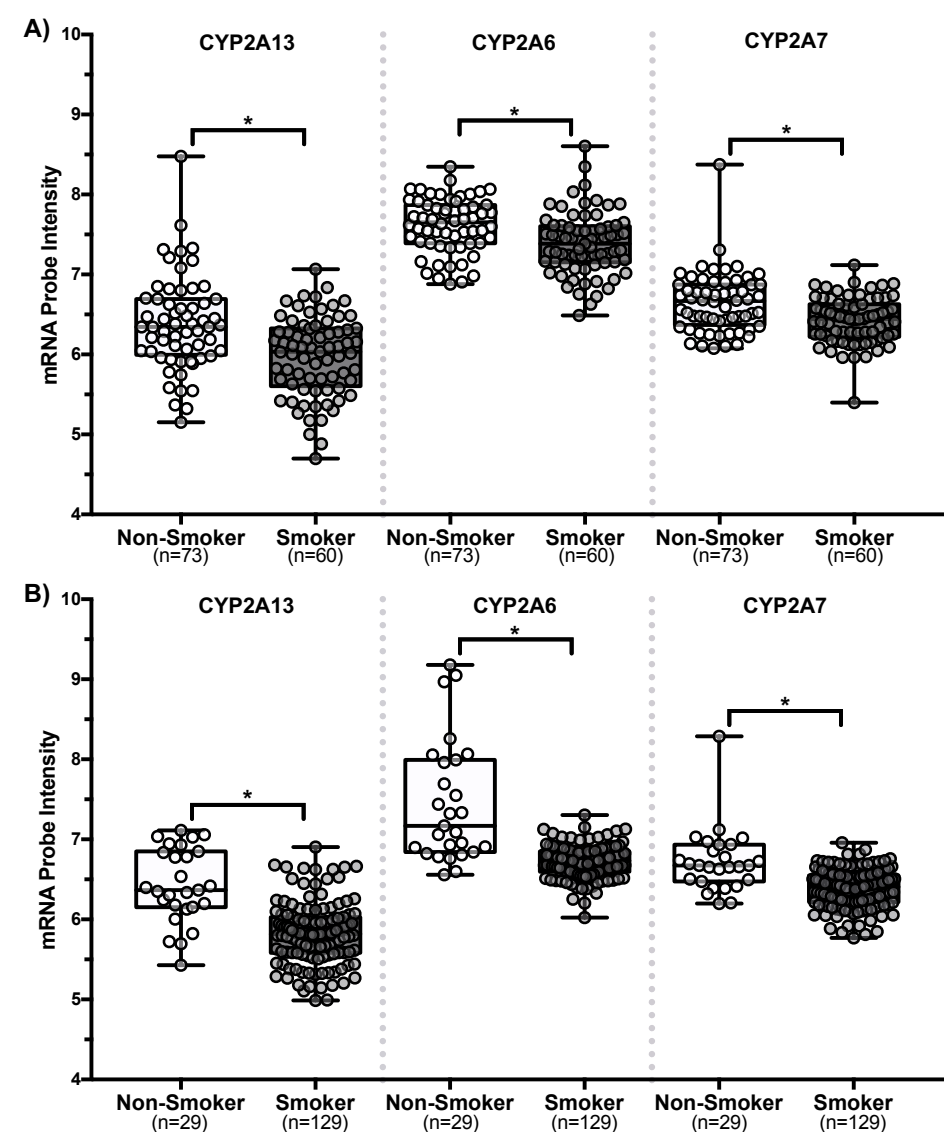

Figure 3. Lung CYP2A13, CYP2A6 and CYP2A7 mRNA levels were lower in smokers than nonsmokers. 
A)
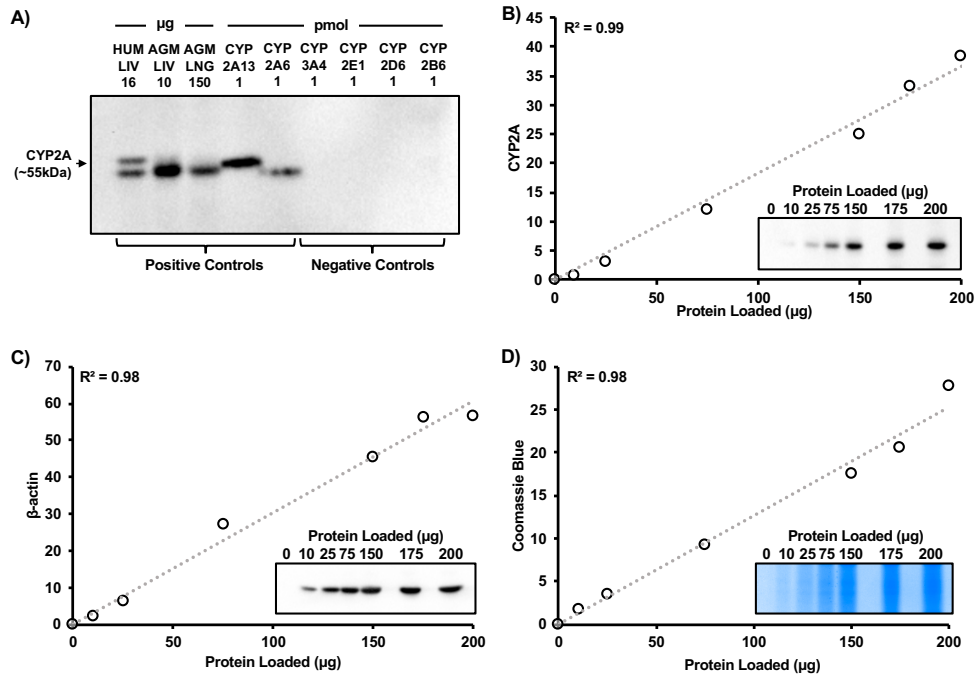

Figure 4. Immunoblotting assays were selective and linear. 

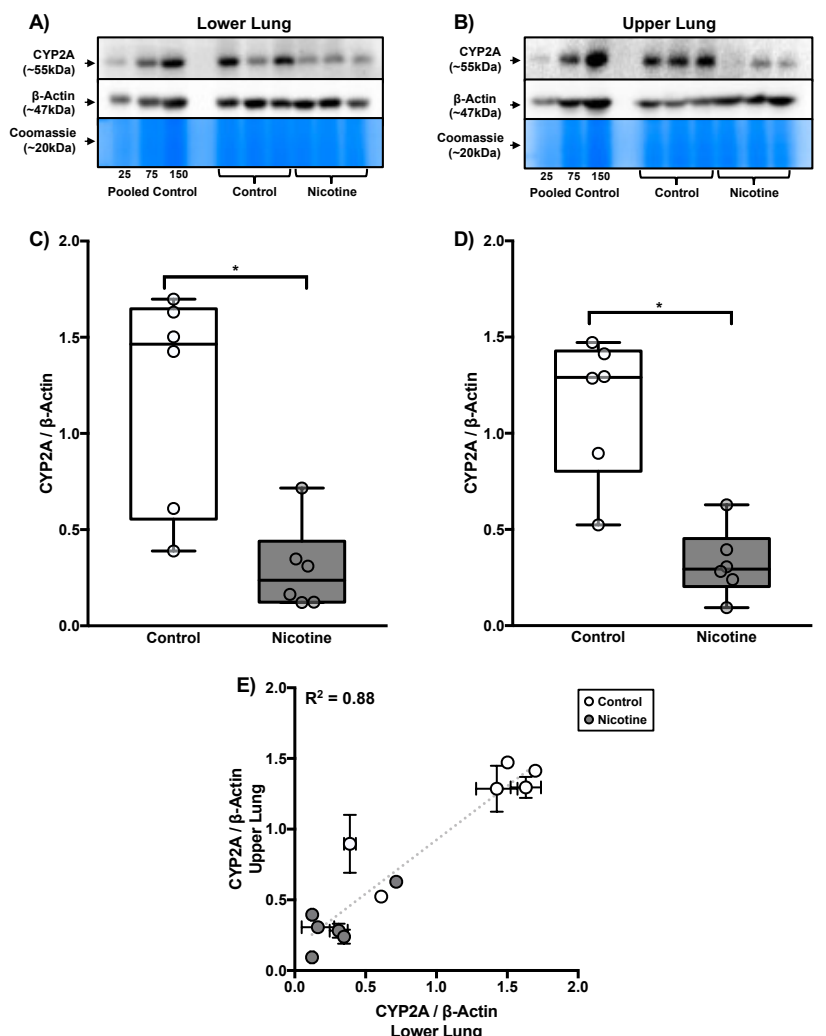

Figure 5. AGM Lung CYP2A protein levels were lower in the Nicotine group compared to the Contro group (AGM Study 1). 

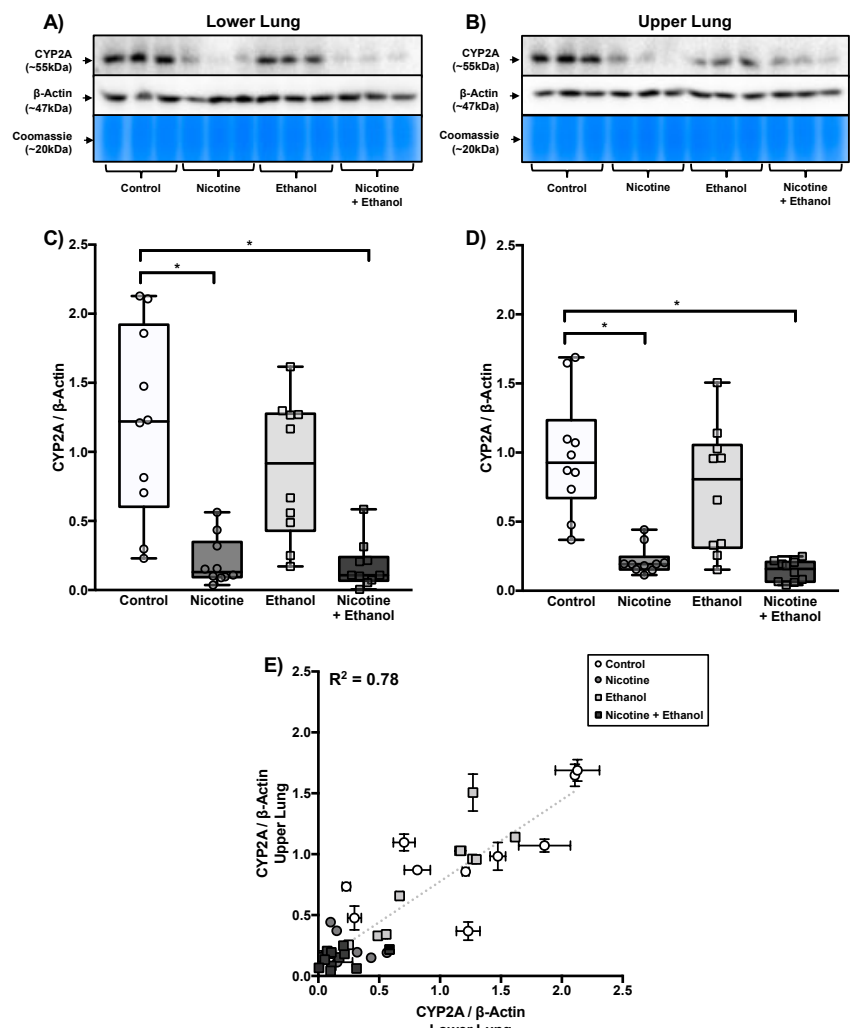

Figure 6. AGM Lung CYP2A protein levels were lower in the Nicotine groups compared to the Control groups with little apparent impact in the Ethanol group (AGM Study 2). 